\title{
Isosteviol prevents the development of isoprenaline-induced myocardial hypertrophy
}

\author{
YAOXU CHEN $^{1}$, HUIMIN BENG $^{1}$, HAO SU$^{1}$, FUPING HAN ${ }^{1}$, ZHUO FAN $^{1}$, \\ NANYING LV ${ }^{1}$, ALEKSANDAR JOVANOVIĆ ${ }^{2,3}$ and WEN TAN ${ }^{1,4}$ \\ ${ }^{1}$ Department of Innovative Drugs and Medicine, School of Bioscience and Bioengineering, South China University \\ of Technology, Guangzhou, Guangdong 510641, P.R. China; ${ }^{2}$ Department of Basic and Clinical Sciences, \\ University of Nicosia Medical School; ${ }^{3}$ Center for Neuroscience and Integrative Brain Research University \\ of Nicosia Medical School, CY-1700 Nicosia, Cyprus; ${ }^{4}$ Institute of Biomedical and Pharmaceutical Sciences, \\ Guangdong University of Technology, Guangzhou, Guangdong 510006, P.R. China
}

Received April 25, 2019; Accepted August 30, 2019

DOI: $10.3892 /$ ijmm.2019.4342

\begin{abstract}
Isosteviol sodium (STVNa), which is a derivate of the natural sweet-tasting glycoside stevioside, has recently been developed and it has been determined that this compound exhibits neuro- and cardio-protective properties. In the current study, whether STVNa interferes with the development of cardiac hypertrophy, which is induced by isoprenaline (Iso), was investigated in an experimental rat model. Rats were treated with a vehicle $(0.9 \% \mathrm{NaCl}$; control), isoprenaline (Iso; $5 \mathrm{mg} / \mathrm{kg}$ ) or Iso $(5 \mathrm{mg} / \mathrm{kg})$ with STVNa $(4 \mathrm{mg} / \mathrm{kg}$; Iso + STVNa). Cardiomyocytes were isolated using enzymatic dissociation and were treated with $5 \mu \mathrm{M}$ Iso for $24 \mathrm{~h}$ and co-treated with $5 \mu \mathrm{M}$ STVNa. Brain natriuretic peptide (BNP) mRNA expression was determined using PCR analysis. Cell surface area, intracellular reactive oxygen species (ROS), mitochondrial transmembrane potential $(\Delta \Psi \mathrm{m})$, cytoplasmic $\mathrm{Ca}^{2+}$ and $\mathrm{Ca}^{2+}$ and contractile function were examined using a laser scanning confocal microscope. The current study demonstrated that STVNa inhibited Iso-induced cardiac hypertrophy by inhibiting cardiomyocyte size. STVNa significantly reduced cell surface area and decreased BNP mRNA expression in ventricular cardiomyocyte Iso-induced hypertrophy. STVNa was also revealed to restore $\Delta \Psi \mathrm{m}$ and reduce ROS generation
\end{abstract}

Correspondence to: Professor Aleksandar Jovanović, Department of Basic and Clinical Sciences, University of Nicosia Medical School, 21 Ilia Papakyriakou, 2414 Engomi, CY-1700 Nicosia, Cyprus

E-mail: jovanovic.a@unic.ac.cy

Professor Wen Tan, Institute of Biomedical and Pharmaceutical Sciences, Guangdong University of Technology, 100 Waihuan West Road, Higher Education Mega Center, Guangzhou, Guangdong 510006, P.R. China

E-mail:went@gdut.edu.cn

Key words: isosteviol, cardiac hypertrophy, reactive oxygen species, mitochondria, $\mathrm{Ca}^{2+}$ and intracellular $\mathrm{Ca}^{2+}$ concentration when compared with the Iso-treated group. Additionally, STVNa preserved $\mathrm{Ca}^{2+}$ transients in hypertrophic cardiomyocytes. In conclusion, the present study demonstrated that STVNa protects against Iso-induced myocardial hypertrophy by reducing oxidative stress, restoring $\Delta \Psi \mathrm{m}$ and maintaining $\mathrm{Ca}^{2+}$ homeostasis.

\section{Introduction}

Stevioside, a natural sweet-tasting glycoside, is found in Stevia rebaudiana. Isosteviol, a derivate of stevioside, has been demonstrated to exhibit a variety of beneficial pharmacological effects (1-5). Isosteviol sodium salt (STVNa), which is a beyerane diterpene, a more soluble and injectable form of isosteviol, has recently been synthesized via acid hydrolysis of stevioside, and it has been determined that STVNa exhibits neuro- and cardio-protective properties (1-5). It has also been indicated that STVNa attenuates right ventricular hypertrophy and pulmonary artery remodeling in an experimental model of transverse aortic constriction and ameliorates diabetic cardiomyopathy $(6,7)$. However, whether STVNa exhibits an effect on the development of left ventricular hypertrophy $(\mathrm{LVH})$ is, to the best of our knowledge, yet to be determined.

$\mathrm{LVH}$ is defined as the enlargement and thickening of the left ventricle walls, which form the main contractile chamber of the heart. $\mathrm{LVH}$ is the ultimate outcome in a variety of pathological states, including hypertension, valvular disease, myocardial infarction and cardiomyopathy (8). This condition is usually associated with the activation of $\beta$-adrenergic signaling and the consequent increase in oxidative stress, protein synthesis, proto-oncogene expression and the stimulation of mitogen activated protein kinases and phosphatidyl inositol-3 kinases (9). The development of pathological LVH is initially beneficial as it allows the heart to maintain its cardiac pump function despite abnormal pressure and/or volume load. However, this ultimately leads to depression of the intrinsic contractile state of the myocardium and subsequent heart failure (8). Additional therapeutic strategies, which prevent LVH and heart failure are urgently required (10). 
Isoprenaline (Iso), a non-selective $\beta$-adrenoceptor agonist, is widely used to induce LVH in animal experimental models of cardiac hypertrophy (11-13). This model successfully mimics sustained adrenergic stimulation, which is a major mechanism in the pathogenesis of maladaptive cardiac hypertrophy (14). In the current study, this particular model was used to assess whether STVNa modifies the development of myocardial hypertrophy and if it does, to determine the underlying mechanism governing this.

\section{Materials and methods}

Materials. All chemicals (including caffeine) used in the current study were purchased from Sigma-Aldrich; Merck $\mathrm{KGaA}$, unless otherwise stated. H2DCFDA, JC-1 and Fluo-4 ester were purchased from Invitrogen; Thermo Fisher Scientific, Inc. mitoTEMPO was purchased from Enzo Life Sciences, Inc. Medium-199 (M199) was purchased from Thermo Fisher Scientific, Inc. PCR reagent kit, primers and markers were purchased from Takara Biotechnology Co., Ltd. STVNa, which is the sodium salt of isosteviol and is a beyerane diterpene, was obtained via acid hydrolysis of stevioside, and was synthesized by the Chemical Synthesis Group of Institute of Biomedical and Pharmaceutical Sciences, Guangdong University of Technology (Guangzhou, China).

Rats and experimental protocol. Sixty male Sprague-Dawley rats (weight, 200-250 g; age, 6 weeks) were obtained from the Experimental Animal Center of Guangzhou University of Chinese Medicine (Guangzhou, China). All animal experimental protocols complied with the Guide for the Care and Use of Laboratory Animals, which was published by the National Institutes of Health. The current study was approved by the Institutional Animal Research Committee of South China University of Technology (Guangzhou, China). Sprague-Dawley rats were housed in a room maintained at $24^{\circ} \mathrm{C}$ and $50 \%$ humidity with a 12 -h light/dark cycle and provided with standard food and water ad libitum. Rats were randomly divided into three groups (60 in total): Control group, treated with vehicle $(0.9 \% \mathrm{NaCl}$; control $)(n=20)$; Iso group, treated with isoprenaline $(5 \mathrm{mg} / \mathrm{kg}$; Iso) $(\mathrm{n}=20)$; Iso + STVNa group, treated with isoprenaline $(5 \mathrm{mg} / \mathrm{kg})$ with isosteviol sodium (4 mg/kg; Iso + STVNa) $(\mathrm{n}=20)$. Vehicle and compounds were injected intraperitoneally daily for 7 days, as previously described (15).

Heart weight index measurement. Rats were weighed (body weight, BW), anesthetized by sodium pentobarbital [intraperitoneal (IP), $50 \mathrm{mg} / \mathrm{kg}$ ] and heparinized (IP, 1,000 U/kg). Rats were sacrificed by overdose of sodium pentobarbital $(>150 \mathrm{mg} / \mathrm{kg})$. The thoracic cavity was subsequently opened and the heart was harvested in a clean glass dish, washed with cold saline solution and weighed [heart weight, (HW)]. The atrium was cut off and the ventricle was separated and weighed [left ventricle weight (LW)]. The tibia length was also measured (Tibia). The heart weight indexes are represented by ratios of HW/BW, HW/Tibia and LW/Tibia.

Histological analysis. Rat hearts were fixed in $10 \%$ formalin at $25^{\circ} \mathrm{C}$ for $8 \mathrm{~h}$. Transverse sections were embedded in paraffin and were cut into $5 \mathrm{~mm}$ sections. Hematoxylin and eosin (H\&E; hematoxylin staining for $5 \mathrm{~min}$, eosin staining for $2 \mathrm{~min}$, at $25^{\circ} \mathrm{C}$ ) were used to assess the cardiomyocyte cross-sectional area. Images were captured with a light microscope and analyzed using ImageJ 1.48 (National Institutes of Health). A total of $>50$ cells were counted in each independent heart from each group.

Isolation of cardiomyocytes and cells treatment. Ventricular myocytes were isolated from untreated, wild-type male Sprague-Dawley rats (200-250 g) as described previously (16), with some modifications. Heparinized (IP; $1,000 \mathrm{U} / \mathrm{kg}$ ) animals were anesthetized using sodium pentobarbital (IP, $50 \mathrm{mg} / \mathrm{kg}$ ). Excised hearts were transferred to a Langendorf perfusion apparatus and perfused with $\mathrm{Ca}^{2+}$-free Tyrode's solution $\left(\mathrm{NaCl} 137 \mathrm{mM}\right.$; $\mathrm{KCl} 5.4 \mathrm{mM} ; \mathrm{NaH}_{2} \mathrm{PO}_{4}$ $1.2 \mathrm{mM} ; \mathrm{MgCl}_{2} .6 \mathrm{H}_{2} \mathrm{O} 1.2 \mathrm{mM}$; HEPES $20 \mathrm{mM}$; taurine $30 \mathrm{mM}$; glucose $20 \mathrm{mM}$; pH 7.4) for $5 \mathrm{~min}$. The perfusion solution was then switched to $\mathrm{Ca}^{2+}$-free Tyrode's solution containing collagenase II $(0.4 \mathrm{mg} / \mathrm{ml})$ and protease (Sigma-Aldrich; Merck KGaA; $0.1 \mathrm{mg} / \mathrm{ml}$ ). After $30 \mathrm{~min}$, ventricles were cut into small pieces, incubated in a $37^{\circ} \mathrm{C}$ water bath and separated into individual cardiomyocytes via slow pipetting. The cells were filtered through a $200 \mathrm{~nm}$ mesh and settled in Tyrode's solution containing $1.2 \mathrm{mM} \mathrm{Ca}^{2+}$ and bovine serum albumin (BSA; 0.1\%). Cells were subsequently re-suspended in M199 (Invitrogen; Thermo Fisher Scientific, Inc.) containing 10\% FBS supplemented with BSA (0.1\%) and transferred to laminin coated culture dishes. After a $1.5 \mathrm{~h}$ of incubation in a $\mathrm{CO}_{2}$ incubator $\left(5 \% \mathrm{CO}_{2} ; 95 \% \mathrm{O}_{2}\right)$, the medium was replaced with serum free M199 (pH 7.4) supplemented with 0.1\% BSA. To induce hypertrophy, cells were treated with $5 \mu \mathrm{M}$ Iso (Sigma-Aldrich; Merck KGaA) for $24 \mathrm{~h}$. To investigate the effect of STVNa on Iso-induced hypertrophy, Iso $(5 \mu \mathrm{M})$-treated cells were co-treated with a variety of STVNa concentrations $(1,5,10$ and $20 \mu \mathrm{M})$. The most effective STVNa concentration $(5 \mu \mathrm{M}$; Fig. S1) was used for subsequent experimentation.

Measurement of ROS, mitochondrial membrane potential and calcium. For ROS measurement, cardiomyocytes were loaded with $10 \mu \mathrm{M} \mathrm{H} 2 \mathrm{DCFDA}$ in serum-free medium at $37^{\circ} \mathrm{C}$ for $20 \mathrm{~min}$ in the dark and then resuspended in $1 \mathrm{mM} \mathrm{Ca}^{2+}$ Tyrode's solution to wash out residues of the dye. DCFDA was excited at $480 \mathrm{~nm}$ and measured at $525 \mathrm{~nm}$. For mitochondrial membrane potential measurement, cardiomyocytes were incubated with $5 \mu \mathrm{M} \mathrm{JC}-1$ at $37^{\circ} \mathrm{C}$ in the dark for $30 \mathrm{~min}$. Cells were washed twice in $1 \mathrm{mM} \mathrm{Ca}^{2+}$ Tyrode's solution. Red fluorescence was exited at $585 \mathrm{~nm}$ and measured at $590 \mathrm{~nm}$. Green fluorescence was exited at $514 \mathrm{~nm}$ and measured at $529 \mathrm{~nm}$. For calcium measurement, cardiomyocytes were exposed to $1 \mu \mathrm{M}$ Fluo- $4 \mathrm{AM}$ at $37^{\circ} \mathrm{C}$ for 40 min for loading and then washed twice in $1 \mathrm{mM} \mathrm{Ca}^{2+}$ Tyrode's solution. Fluo- 4 was excited at $488 \mathrm{~nm}$ and measured at $>520 \mathrm{~nm}$. For analysis, intensity of fluorescence for targeted cells was directly read using the Zeiss LSM 710 confocal software (ZEN version 2011, Carl Zeiss Meditec AG).

Measurement of cell surface area. Phase contrast images that were captured using a light Olympus IX83 microscope (Olympus Corporation) were used to measure the surface 
A

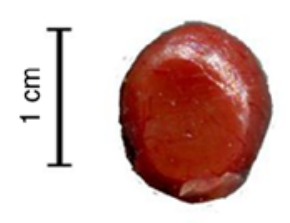

Con

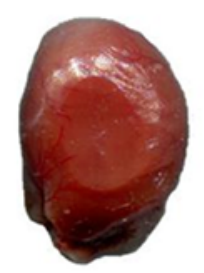

Iso

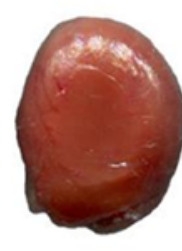

Iso+STVNa
B
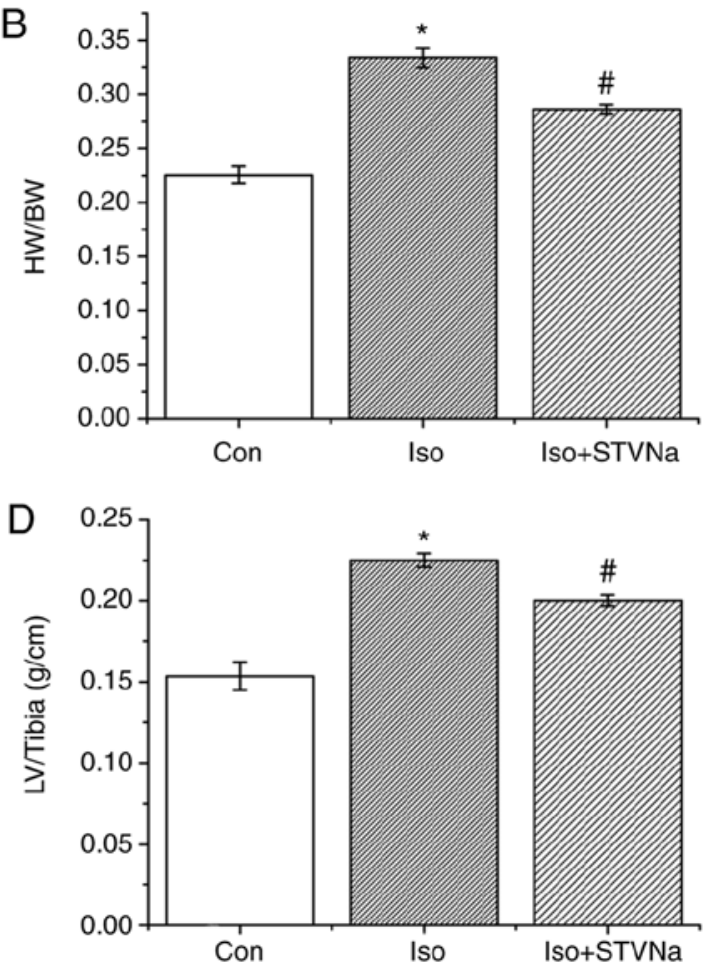

Figure 1. STVNa prevents Iso-induced cardiac hypertrophy. (A) Original images of hearts from different experimental groups as labelled. Graphs depicting (B) HW/Tibia (C) HW/BW and (D) LV/Tibia. Each bar represents the mean \pm standard error of the mean. ${ }^{*} \mathrm{P}<0.05$ vs. the Con group; ${ }^{\text {P }}<0.05$ vs. Iso group ( $\mathrm{n}=6$ for each). HW, heart weight; Tibia, tibia length; BW, body weight; LV, left ventricular weight; Iso, isoprenaline; STVNa, isosteviol sodium; Con, control.

area of different groups using Image Pro-Plus 6.0 (National Institutes of Health) software. A total of 120 cells from six different animals were analyzed to determine the morphological changes that were induced by Iso.

Measurement of mRNA levels. The effect of STVNa on the hypertrophic response of cardiomyocytes to Iso stimulus was assessed by monitoring BNP mRNA expression using reverse transcription (RT)-quantitative (q) PCR. Total RNA was extracted from cells using RNAiso Plus (Takara Biotechnology Co., Ltd.; Total RNA extraction reagent) according to the manufacturer's protocol. The concentration was determined by measuring the absorbance at $260 \mathrm{~nm}$ and RNA purity was determined by measuring 260/280 ratio using a NanoDrop 2000c Spectrophotometer (Thermo Fisher Scientific, Inc.). Total RNA $(0.5 \mu \mathrm{g})$ was used for RT with the PrimeScript II First Strand cDNA synthesis kit (cat. no. 6210A; Takara Biotechnology Co., Ltd.), following the manufacturer's protocol. qPCR was performed by using ChamQ SYBR PCR kit Q311-01 (Vazyme Biotech Co., Ltd.). Relative quantification of gene expression was normalized to GAPDH. The nucleotide sequences of the primers used were: BNP forward, 5'-CTGTGACGGGCT GAGGTT-3' and reverse, 5'-GCAAGTTTGTGCTGGAAG-3'; GAPDH forward, 5'-GCAAGTTCAACGGCACAG-3', and reverse, 5'-CGCCAGTAGACTCCACGAC-3'.

Analysis of cardiomyocyte contractile function. Cell contraction was recorded in the frame-scanning mode and time-series mode using a Zeiss LSM 710 confocal microscope. Cells were stimulated to contract at $1 \mathrm{~Hz}$ and scanned for $5 \mathrm{~min}$ (100 ms/Frame). The rate of contraction and shortening were measured and analyzed using the Zeiss LSM Imaging processing software (ZEN version 2011; Carl Zeiss Meditec AG).

Statistical analysis. Data are expressed as the mean \pm standard error of the mean from three experimental repeats. Statistical analysis was performed using a one-way analysis of variance followed by the Tukey post-hoc test (SigmaPlot v14; Jandel Corporation). $\mathrm{P}<0.05$ was considered to indicate a statistically significant difference. For the analysis of HW/BW, cross-sectional area of $\mathrm{H} \& \mathrm{E}$ staining, cell surface area of cardiomyocytes, BNP mRNA expression, ROS fluorescence intensity and $\mathrm{Ca}^{2+}$ fluorescence intensity, the raw mean value of the control group was used as a reference value, and the raw value of the control group was divided by this value. The raw mean value of the control group was set at 1 and the data of the other groups were presented as the fold-change of the control.

\section{Results}

STVNa prevents the development of Iso-induced cardiac hypertrophy. Experiment were carried out using rat heart tissues. Treatment with Iso significantly increased HW/BW $(1.00 \pm 0.02$ vs. $1.53 \pm 0.05 ; \mathrm{P}<0.05 ; \mathrm{n}=6 ;$ Fig. 1$), \mathrm{HW} /$ tibia $(0.23 \pm 0.01 \mathrm{~g} / \mathrm{cm}$ vs. $0.33 \pm 0.01 \mathrm{~g} / \mathrm{cm} ; \mathrm{P}<0.05 ; \mathrm{n}=6 ;$ Fig. 1) and LV/tibia $(0.15 \pm 0.01 \mathrm{~g} / \mathrm{cm}$ vs. $0.23 \pm 0.01 \mathrm{~g} / \mathrm{cm} ; \mathrm{P}<0.05$; $\mathrm{n}=6$; Fig. 1) ratios. This effect was inhibited by STVNa (Fig. $1 ; \mathrm{HW} / \mathrm{BW}, 1.53 \pm 0.05$ vs. $1.35 \pm 0.06 ; \mathrm{n}=6 ; \mathrm{P}=0.05$; HW/tibia, $0.33 \pm 0.01 \mathrm{~g} / \mathrm{cm}$ vs. $0.29 \pm 0.01 \mathrm{~g} / \mathrm{cm} ; \mathrm{n}=6 ; \mathrm{P}<0.05$; LV/tibia, $0.23 \pm 0.01 \mathrm{~g} / \mathrm{cm}$ vs. $0.20 \pm 0.01 \mathrm{~g} / \mathrm{cm} ; \mathrm{n}=6 ; \mathrm{P}=0.01$ ). The histological analysis of myocardial tissues demonstrated 
A

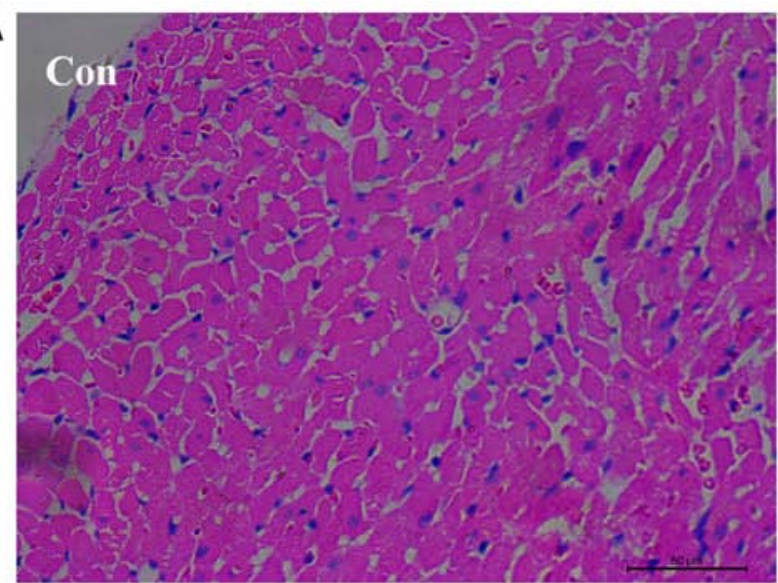

B

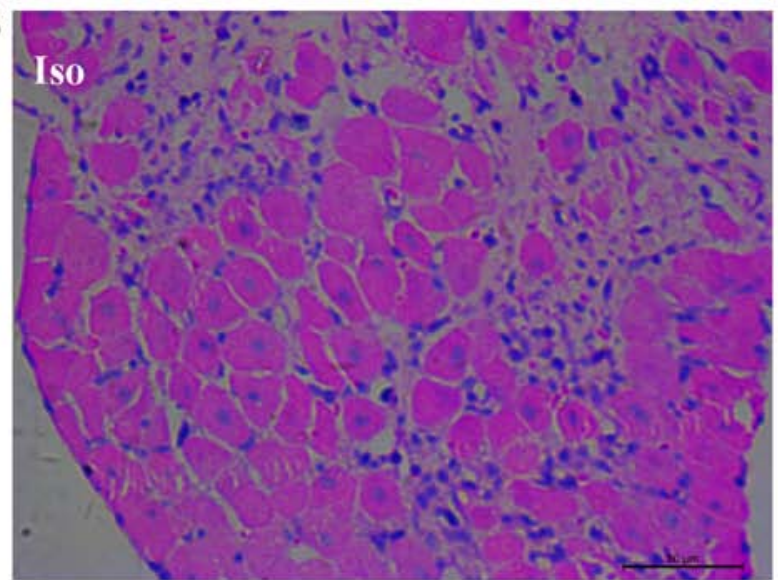

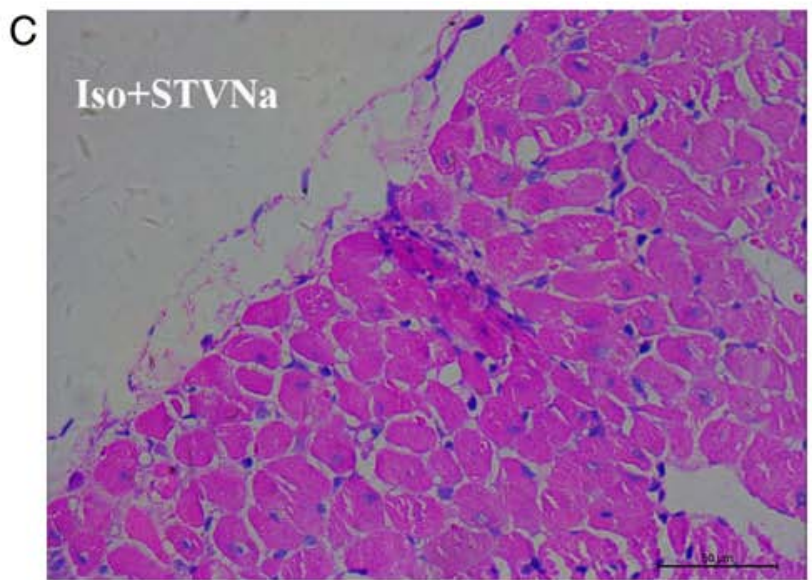

D

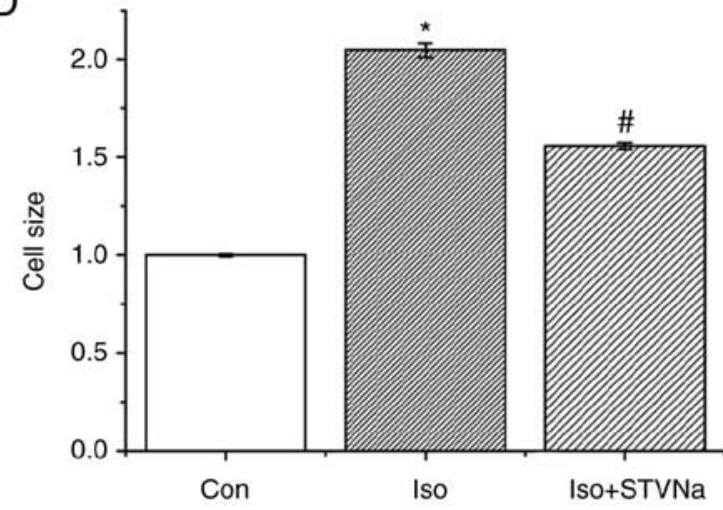

Figure 2. STVNa prevents Iso-induced increase in cardiomyocyte size. Experiment were carried out using rat cardiac tissue. Original images of hematoxylin and eosin staining from the left ventricle (Scale bar=50 $\mu \mathrm{m}$ ) in (A) Con group, (B) Iso group and (C) Iso + STVNa group. (D) Graph corresponding to the conditions in A, B and C. Each bar represents the mean \pm standard error of the mean. ${ }^{*} \mathrm{P}<0.05$ vs. the Con group; ${ }^{\text {"P }}<0.05$ vs. Iso group ( $\mathrm{n}=6$ for each). Iso, isoprenaline; STVNa, isosteviol sodium; Con, control.

that cardiomyocyte cross sectional areas were significantly increased in mice treated with Iso $(1.00 \pm 0.01$ fold-change vs. $2.05 \pm 0.04$ fold-change; $n=6 ; P<0.05$; Fig. 2 ) and this increase was partly inhibited by STVNa (1.56 \pm 0.02 fold-change; $n=6$; $\mathrm{P}>0.05$ vs. control; Fig. 2). Similar results were obtained subsequent to the examination of the effects of Iso and STVNa treatments on cardiomyocytes size in vitro, which were carried out using cardiomyocytes isolated from rats. Although STVNa did not solely affect cardiomyocyte surface area (control, $1.01 \pm 0.05$ fold-change; STVNa, $1.04 \pm 0.03$ fold-change, $n=7$ for each; $\mathrm{P}=0.639$; Fig. 3) it prevented an increase in this parameter that was induced by Iso (Iso, $1.39 \pm 0.04$ fold-change; Iso + STVNa, $1.15 \pm 0.03$ fold-change; $\mathrm{n}=7$ for each; $\mathrm{P}<0.05$; Fig. 3).

BNP mRNA is a well-established biomarker for cardiac hypertrophy and heart failure (17). mRNA measurement was carried out using cardiomyocytes isolated from untreated, wild-type rats. Iso treatment significantly increased BNP mRNA levels in cardiomyocytes (control, 1.00 \pm 0.10 fold-change; Iso, $2.25 \pm 0.25$ fold-change; $\mathrm{n}=5$ for each; $\mathrm{P}<0.05$; Fig. 4). STVNa prevented this increase ( $1.61 \pm 0.25$ fold-change; $\mathrm{n}=5 ; \mathrm{P}<0.05$ vs. control; Fig. 4) although it did not exhibit any effect on BNP mRNA levels when used on its own $(1.07 \pm 0.08$ fold-change; $n=5 ; \mathrm{P}>0.05$ vs. control; Fig. 4).
STVNa reduces ROS production in cardiomyocytes treated with Iso. Iso treatment significantly increased ROS production in cardiomyocytes as indicated by DCFH fluorescence (from $1.00 \pm 0.06$ fold-change under control conditions to $2.04 \pm 0.07$ fold-change when treated with Iso; $n=6$ for each; $\mathrm{P}<0.001$; Fig. 5). STVNa did not solely affect ROS production $(1.09 \pm 0.05$ fold-change; $n=6 ; P=0.524$ vs. the control; Fig. 5). However, STVNa prevented the effect exhibited by Iso $(1.52 \pm 0.13$ fold-change; $n=6 ; \mathrm{P}=0.004$ vs. Iso-treated group; Fig. 5) in a similar manner to mitoTEMPO, a mitochondrial-targeted antioxidant $(1.72 \pm 0.13$ fold-change; $\mathrm{n}=6 ; \mathrm{P}=0.274$ vs. Iso + STVNa-treated group; Fig. 5). Experiments were carried out using cardiomyocytes isolated from untreated, wild-type rats.

STVNa prevents mitochondrial membrane depolarization induced by Iso treatment. Treatment with Iso led to mitochondrial membrane depolarization in wild-type cardiomyocytes (isolated from untreated wild-type rats) as indicated by the significant decrease that was observed in channels ratio (from $2.49 \pm 0.20$ under control conditions to $1.53 \pm 0.03$ following Iso treatment; $n=6$ for each; $\mathrm{P}<0.05$; Fig. 6). STVNa did not solely affect mitochondrial membrane potential $(2.56 \pm 0.20$; $\mathrm{n}=6 ; \mathrm{P}=0.746$ vs. control; Fig. 6). However, STVNa inhibited 




Con

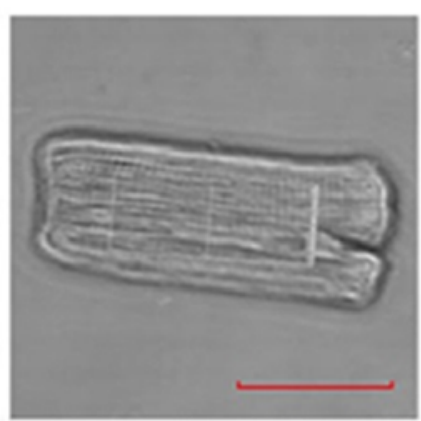

Iso

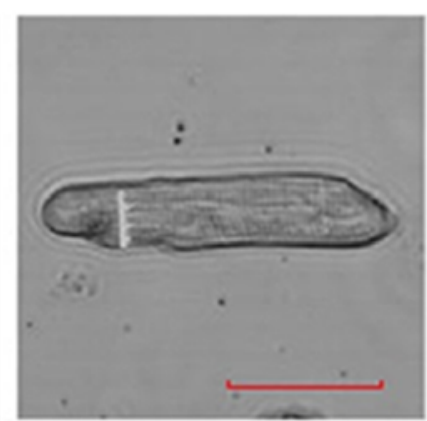

Iso+STVNa

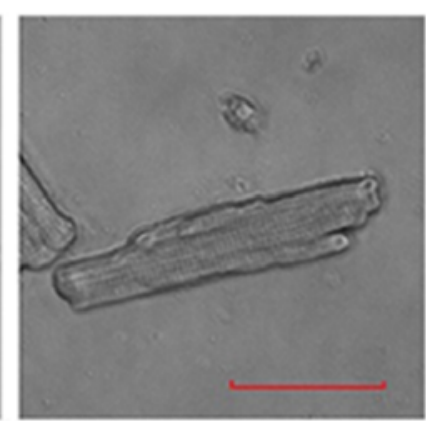

STVNa

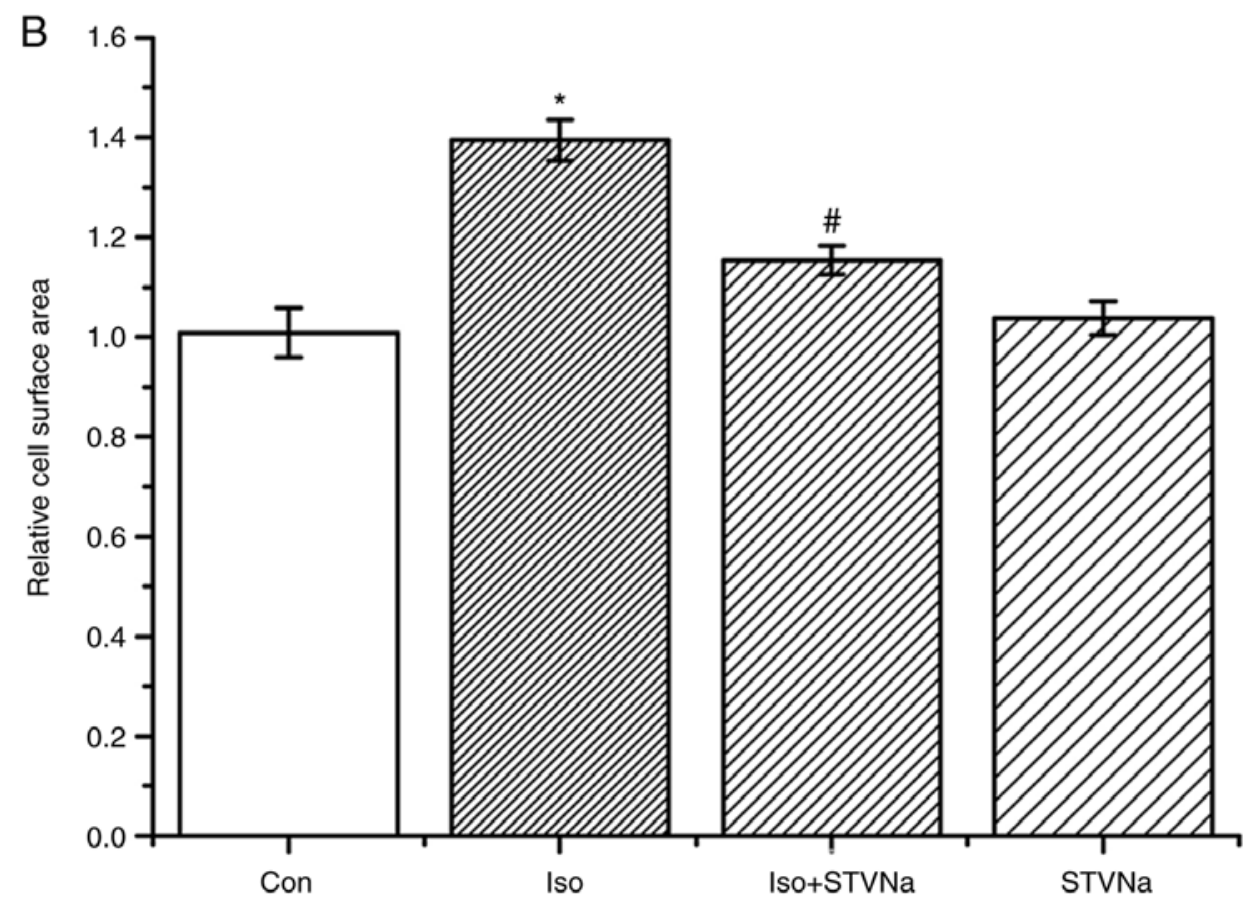

Figure 3. STVNa prevents the increase in cardiomyocyte surface area induced by Iso treatment in vitro. Experiments were carried out using cardiomyocytes isolated from rats. Original images of cardiomyocytes under (A) labelled conditions and the (B) corresponding graph. Each bar represents mean \pm standard error of the mean. " $\mathrm{P}<0.05$ vs. the Con group; ${ }^{\#} \mathrm{P}<0.05$ vs. Iso group ( $\mathrm{n}=7$ for each). Iso, isoprenaline; STVNa, isosteviol sodium; Con, control.

A

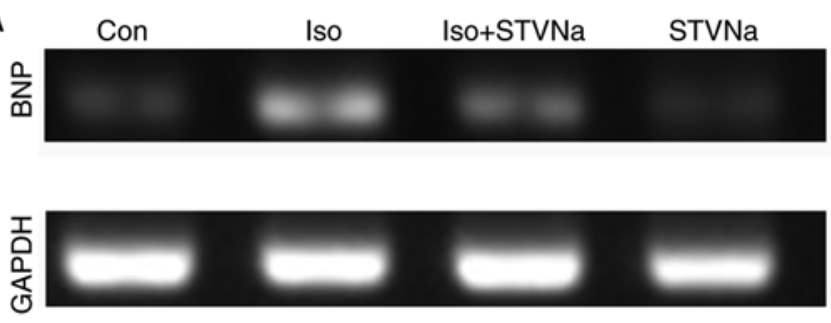

B

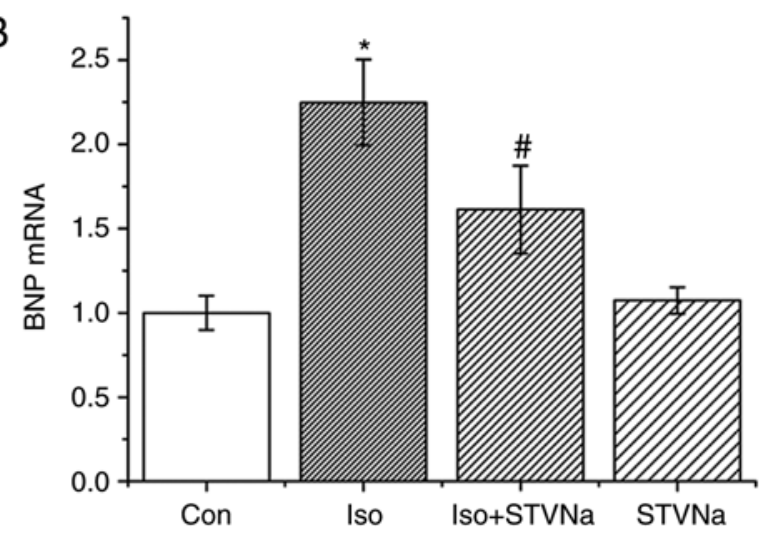

Figure 4. STVNa prevents the increase in cardiomyocytes BNP mRNA level induced by Iso treatment. Experiments were carried out using cardiomyocytes isolated from rats. Original images of reverse transcription PCR products under (A) labelled conditions and the (B) corresponding graph. Each bar represents the mean \pm standard error of the mean. ${ }^{*} \mathrm{P}<0.05$ vs. the Con group; ${ }^{\text {}} \mathrm{P}<0.05$ vs. Iso group ( $\mathrm{n}=5$ for each). BNP, brain natriuretic peptide; Iso, isoprenaline; STVNa, isosteviol sodium; Con, control.

the effect of Iso treatment $(1.89 \pm 0.11 ; \mathrm{n}=6 ; \mathrm{P}=0.011$ vs. Iso group; Fig. 6).
STVNa prevents $\mathrm{Ca}^{2+}$ loading and impaired $\mathrm{Ca}^{2+}$ dynamics induced by Iso treatment. Cardiomyocytes were isolated from 


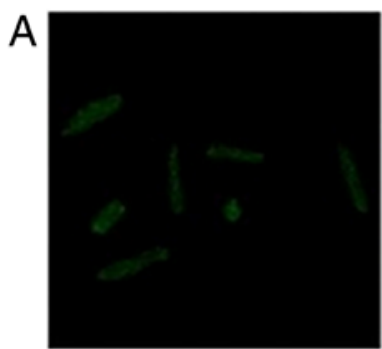

Con

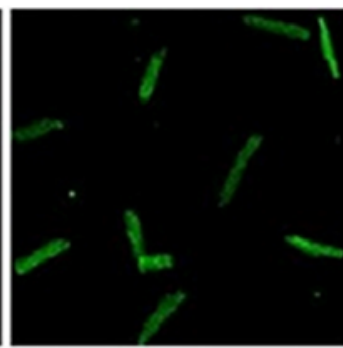

Iso

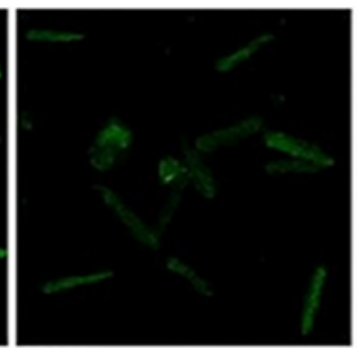

Iso+STVNa

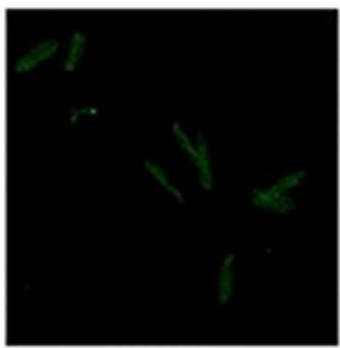

Iso+Mito TEMPO

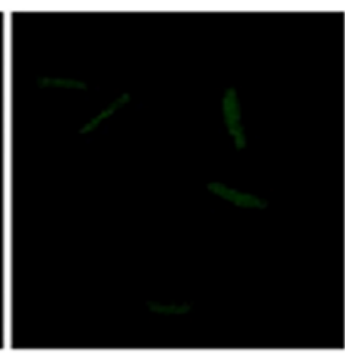

STVNa

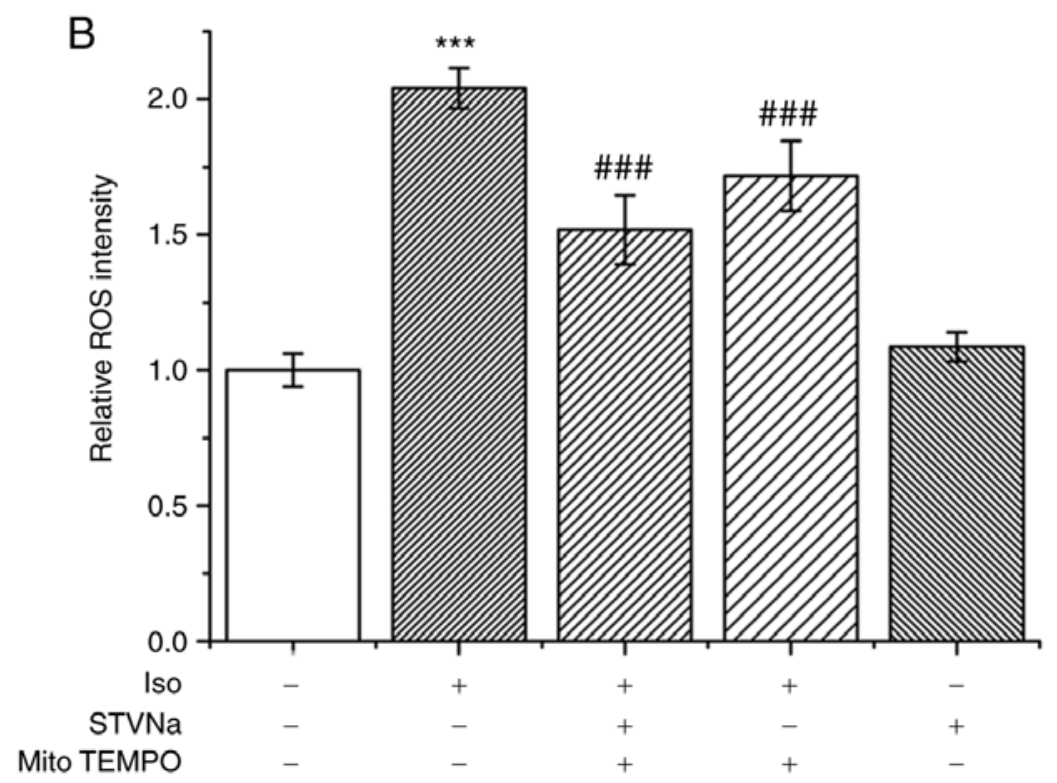

Figure 5. STVNa reduces ROS production in cardiomyocytes treated with Iso. Experiments were carried out using cardiomyocytes isolated from rats. Original images under (A) labelled conditions and the (B) corresponding graph. The level of ROS was detected using DCF. Scale bar is $100 \mu \mathrm{m}$. Each bar represents the mean \pm standard error of the mean. ${ }^{* * *} \mathrm{P}<0.01$ vs. Con group; ${ }^{\# \# ~} \mathrm{P}<0.01$ vs. Iso group ( $\mathrm{n}=6$ for each). ROS, reactive oxygen species; Iso, isoprenaline; STVNa, isosteviol sodium; Con, control.

A JC1-

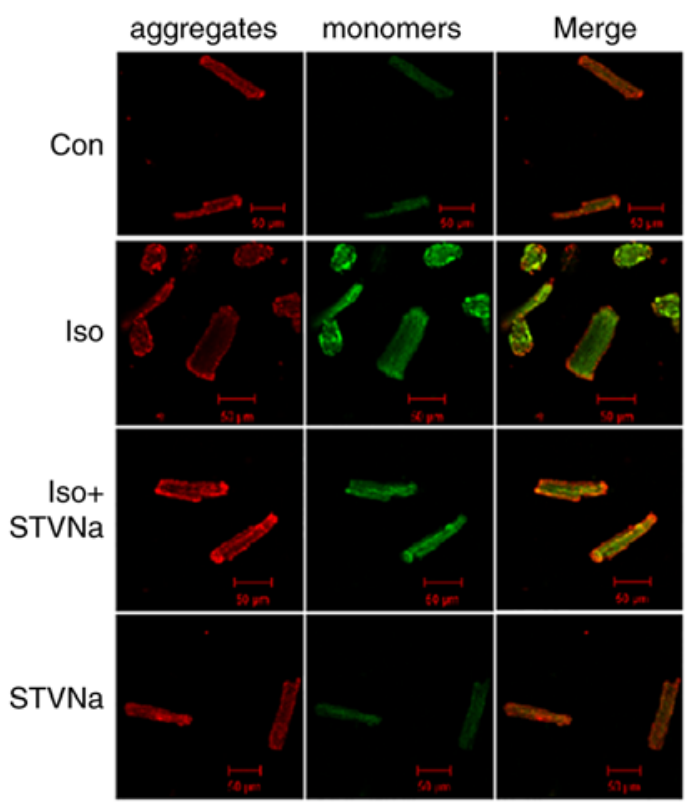

B

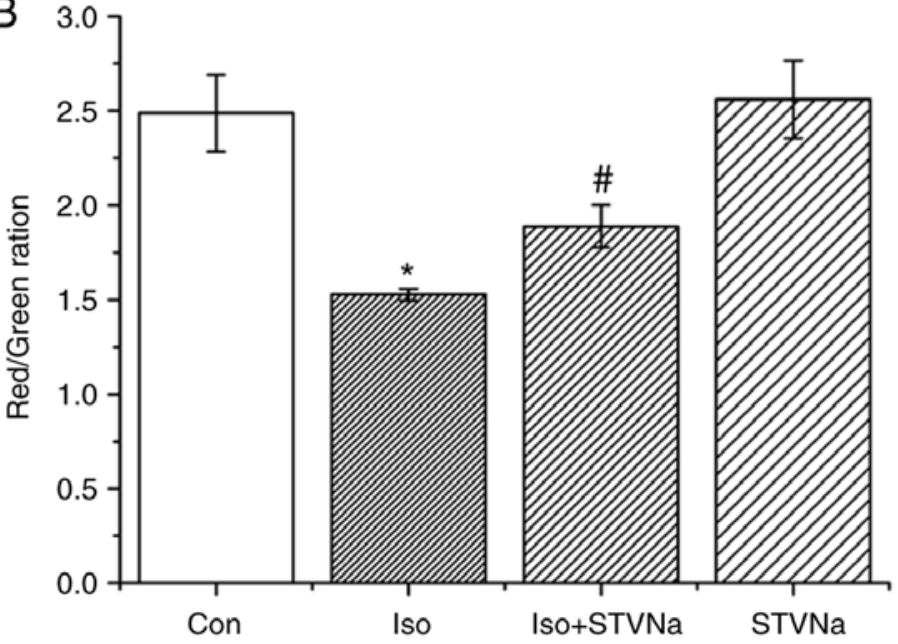

Figure 6. STVNa prevents mitochondrial membrane depolarization induced by Iso treatment. Experiments were carried out using cardiomyocytes isolated from rats. Original images under (A) labelled conditions and the (B) corresponding graph. Mitochondrial membrane potential was measured using JC-1. Scale bar is $100 \mu \mathrm{m}$. Each bar represents the mean \pm standard error of the mean. ${ }^{*} \mathrm{P}<0.05 \mathrm{vs}$. Con; ${ }^{\#} \mathrm{P}<0.05 \mathrm{vs}$. Iso group ( $\mathrm{n}=6$ for each). Iso, isoprenaline; $\mathrm{STVNa}$, isosteviol sodium; Con, control. 



Figure 7. STVNa prevents $\mathrm{Ca}^{2+}$ loading that is induced by Iso treatment. Experiments were carried out using cardiomyocytes isolated from rats. Original images captured by the frame-scanning mode of the confocal microscope under (A) labelled conditions and the (B) corresponding graph. The level of $\mathrm{Ca}^{2+}$ was detected using Fluo-4. Scale bar is $50 \mu \mathrm{m}$. Each bar represents the mean \pm standard error of the mean. ${ }^{*} \mathrm{P}<0.05$ vs. the Con group; ${ }^{\#} \mathrm{P}<0.05$ vs. Iso group ( $\mathrm{n}=6$ for each). Iso, isoprenaline; STVNa, isosteviol sodium; Con, control.

untreated wild-type rats. Treatment with Iso induced intracellular $\mathrm{Ca}^{2+}$ loading as reflected by the significant increase observed in Fluo-4 fluorescence (from 1.02 \pm 0.07 fold-change under control conditions to $1.66 \pm 0.11$ fold-change when treated with Iso; $\mathrm{n}=6$ for each; $\mathrm{P}<0.05$; Fig. 7). STVNa did not solely affect intracellular $\mathrm{Ca}^{2+}(1.07 \pm 0.06$ fold-change; $\mathrm{n}=6$; $\mathrm{P}=0.707$ vs. control; Fig. 7). However, STVNa inhibited the effect of Iso treatment $(1.31 \pm 0.15$ fold-change; $n=6 ; P=0.04$ vs . Iso group alone; Fig. 7).

To examine any potential changes in $\mathrm{Ca}^{2+}$ dynamics, the transient $\mathrm{Ca}^{2+}$ was assessed in cardiomyocytes. The amplitude of calcium transient (F/F0) significantly decreased (from 2.64 \pm 0.25 under control conditions to $1.67 \pm 0.07$ when treated with Iso; $n=9$ for each; $P=0.013$; Fig. 8) and the time of $\mathrm{Ca}^{2+}$ uptake was significantly extended (T50 values were $0.29 \pm 0.02 \mathrm{sec}$ under control conditions and $0.38 \pm 0.02 \mathrm{sec}$ when treated with Iso; $n=9$ for each; $\mathrm{P}=0.006$; Fig. 8). Co-treatment with STVNa prevented the effects of Iso treatment $(\mathrm{F} / \mathrm{F} 0$, $2.67 \pm 0.30 ; \mathrm{n}=8 ; \mathrm{P}=0.860$ vs. control; T50, $0.28 \pm 0.02 \mathrm{~S} ; \mathrm{n}=9$; $\mathrm{P}=0.711$ vs. control; Fig. 8).

STVNa prevents the sarcoplasmic reticulum (SR) $\mathrm{Ca}^{2+}$ depletion that is induced by Iso. Caffeine (10 $\mathrm{mM})$ was used to measure the quantities of $\mathrm{Ca}^{2+}$ stored in SR. Iso significantly decreased the quantity of $\mathrm{Ca}^{2+}$ in SR (from $12.65 \pm 0.42$ under control conditions to $8.03 \pm 0.55$ when treated with Iso; $\mathrm{n}=6$ for each; $\mathrm{P}<0.05$; Fig. 9). STVNa inhibited this effect $(10.30 \pm 0.43$; $\mathrm{n}=6 ; \mathrm{P}=0.004$ vs. the Iso group; Fig. 9).

STVNa prevents the Iso-induced impairment of cardiomyocytes contractile function. Treatment with Iso decreased shortening and rate of contraction of wild-type cardiomyoctyes (shortening, from $6.76 \pm 0.67 \%$ under control conditions to $5.13 \pm 0.30 \%$ when treated with Iso; $n=8$ for each; $P=0.067$; rate of contraction, from $0.28 \pm 0.03 \% . \mathrm{S}^{-1}$ under control conditions to $0.17 \pm 0.02 \% . \mathrm{S}^{-1}$ when treated with Iso; $\mathrm{n}=8$ for each; $\mathrm{P}=0.009$; Fig. 10). STVNa inhibited both effects exhibited by Iso treatment (shortening, $7.81 \pm 0.49 \% ; \mathrm{n}=8 ; \mathrm{P}=0.004$ vs. Iso; rate of contraction, $0.30 \pm 0.02 \% . \mathrm{S}^{-1} ; \mathrm{n}=8 ; \mathrm{P}=0.003$ vs. Iso; Fig. 10).

\section{Discussion}

A chronic increase in sympathetic activation occurs during hypertension, obesity, sleep apnea and mental stress, and this can promote the development of cardiac hypertrophy and heart failure through the sustained stimulation of $\beta$-adrenergic receptors (18). The results of the current study demonstrated that sustained stimulation with Iso induces cardiac hypertrophy, which is in agreement with the well-established features of the 

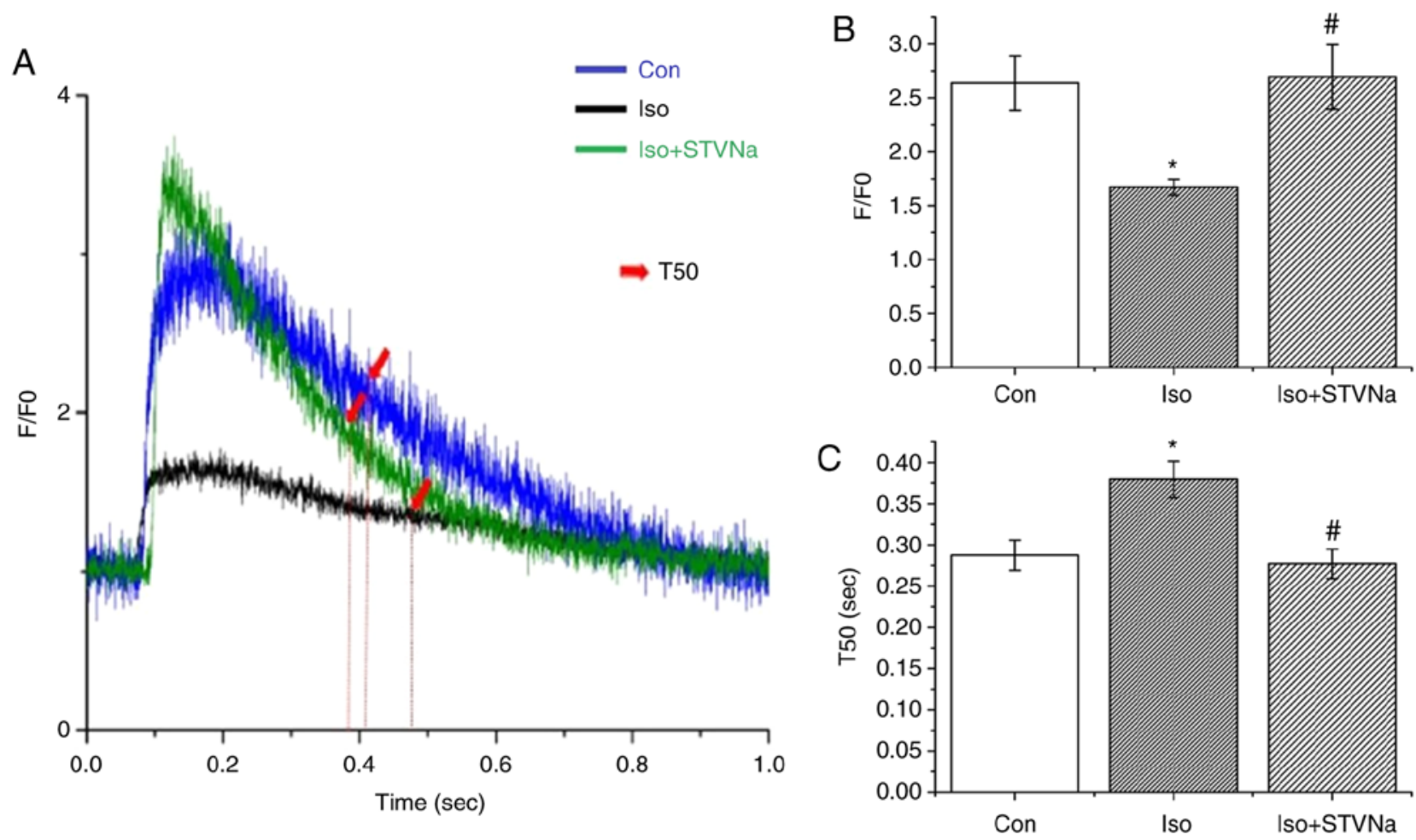

Figure 8. STVNa prevents impairment in $\mathrm{Ca}^{2+}$ dynamics induced by Iso treatment. Experiments were carried out using cardiomyocytes isolated from rats. (A) Original tracing of $\mathrm{Ca}^{2+}$ transients. Intracellular $\mathrm{Ca}^{2+}$ was monitored using Fluo-4. Bar graph showing (B) F/F0 and (C) T50 for the groups corresponding in A. Each bar represents the mean \pm standard error of the mean. ${ }^{*} \mathrm{P}<0.05$ vs. the Con group; ${ }^{~} \mathrm{P}<0.05$ vs. Iso group ( $\mathrm{n}=9$ for each). Iso, isoprenaline; STVNa, isosteviol sodium; Con, control.
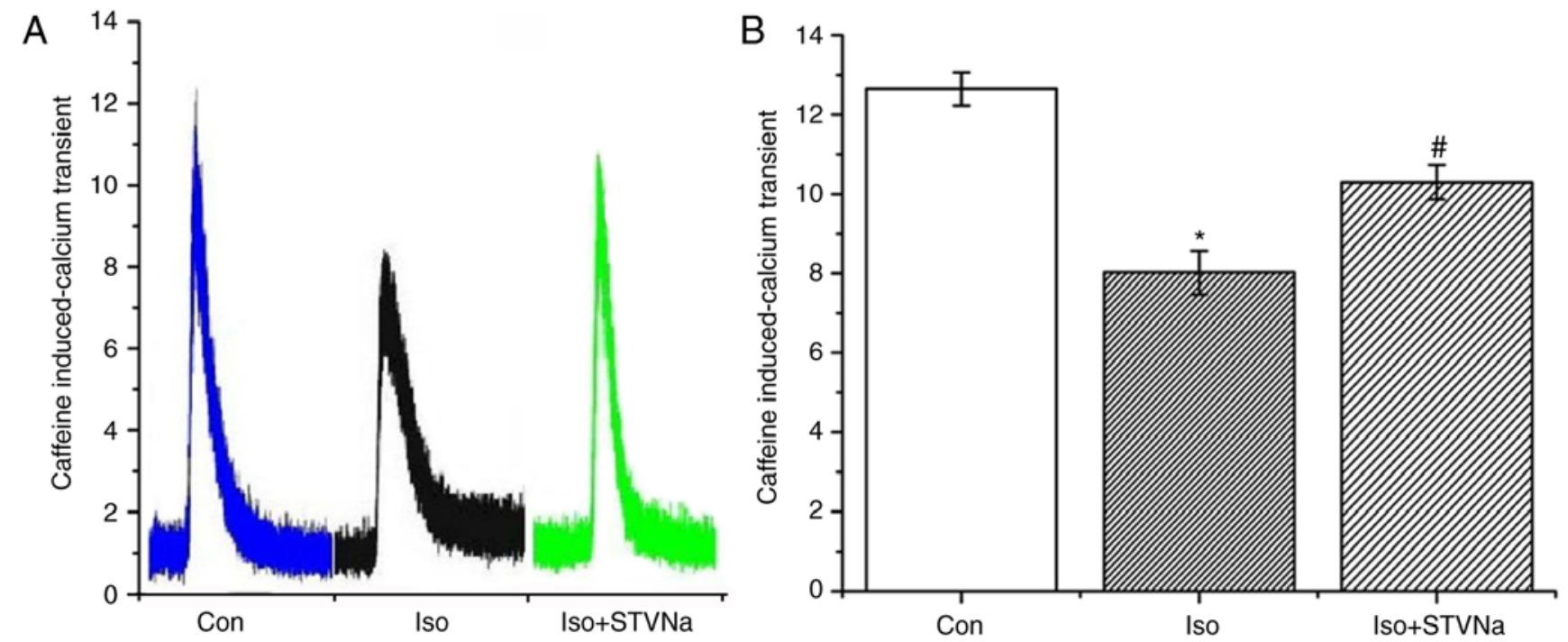

Figure 9. STVNa prevents SR calcium depletion induced by Iso. Caffeine induced $\mathrm{Ca}^{2+}$ transients indicating $\mathrm{Ca}^{2+}$ content of SR. Experiments were carried out using cardiomyocytes isolated from rats. Representative traces of Fluo-4 fluorescence following application of (A) caffeine with the (B) corresponding graph. Each bar represents the mean \pm standard error of the mean. ${ }^{*} \mathrm{P}<0.05$ vs. the Con group; ${ }^{*} \mathrm{P}<0.05$ vs. Iso group ( $\mathrm{n}=6$ for each). Iso, isoprenaline; $\mathrm{STVNa}$, isosteviol sodium; Con, control; SR, sarcoplasmic reticulum.

experimental model used (11). The current study revealed that the induction of cardiac hypertrophy by Iso was associated with i) increased ROS, ii) mitochondrial membrane depolarization, iii) intracellular $\mathrm{Ca}^{2+}$ loading, iv) impaired $\mathrm{Ca}^{2+}$ transients and v) impaired cardiac contractility.

A central mechanism that is associated with the development of cardiac hypertrophy is an increase in ROS and the subsequent oxidative stress (19). The activation of $\beta$-adrenoreceptors has been specifically linked with ROS generation and cardiac hypertrophy $(20,21)$. Oxidative stress has been indicated to activate extracellular signal regulated kinase $1 / 2$ and stimulate protein synthesis in ventricular remodeling $(13,22)$. It has also been suggested that compounds attenuating oxidative stress may also attenuate cardiac hypertrophy (23). In the present 



Figure 10. STVNa prevents Iso-induced impairment of cardiomyocytes contractile function. Experiments were carried out using cardiomyocytes isolated from rats. Bar graphs depicting (A) rate of contraction $(\% / \mathrm{S})$ and $(\mathrm{B})$ shortening of cardiomyocytes $(\%)$. Each bar represents the mean \pm standard error of the mean. ${ }^{*} \mathrm{P}<0.05$ vs. the Con group; ${ }^{~} \mathrm{P}<0.05$ vs. Iso group ( $\mathrm{n}=8$ for each). Iso, isoprenaline; STVNa, isosteviol sodium; Con, control.

study, it was demonstrated that STVNa did not solely affect ROS levels, but prevented an Iso-induced increase in ROS, making this compound a potential therapeutic candidate for use in the prevention of cardiac hypertrophy.

In addition to ATP synthesis, the electron transport chain of mitochondria is a significant source of ROS (24), which, in turn, can damage mitochondria and affect the activity and function of mitochondrial ion channels. Any alterations in mitochondrial ion channel function and mitochondrial homeostasis is reflected in the mitochondrial membrane potential. Mitochondrial membrane depolarization is a well-established indicator of disturbed mitochondrial homeostasis (25). The results of the current study indicated that Iso-treatment induced mitochondrial membrane depolarization, which is supported by previous studies (26-28) that have used this experimental model. STVNa did not solely affect mitochondrial membrane potential but prevented the mitochondrial membrane depolarization that was induced by Iso. These results suggested that STVNa prevented increases in ROS and consequently, mitochondrial damage.

Intracellular $\mathrm{Ca}^{2+}$ homeostasis is crucial for cardiac contractile function (29). Intracellular $\mathrm{Ca}^{2+}$ levels have been demonstrated to reflect the overall metabolic condition of cardiomyocytes $(30,31)$. In the present study, Iso-pretreatment was revealed to increase intracellular $\mathrm{Ca}^{2+}$ and impair intracellular $\mathrm{Ca}^{2+}$ transients and cardiomyocytes contractility. These effects exhibited by Iso were expected, due to the results of multiple studies that indicated that sustained stimulation with $\beta$-agonists increased intracellular $\mathrm{Ca}^{2+}$ levels and impaired contractility (32-34). The sustained increase in $\mathrm{Ca}^{2+}$ activated the protein phosphatase calcineurin and its target, the NFAT family of transcription factors, which are critical mediators of pathological hypertrophy (35). Links between mitochondrial impairment, intracellular $\mathrm{Ca}^{2+}$ loading, impaired contractility and cardiac hypertrophy resulting in heart failure are well established (36). STVNa was revealed to prevent all negative events associated with sustained activation of $\beta$-adrenoceptors, including cardiac hypertrophy, ROS production, mitochondrial membrane depolarization, impaired $\mathrm{Ca}^{2+}$ homeostasis and cardiomyocytes contractility.

A recent study has demonstrated that STVNa sensitizes ATP-sensitive $\mathrm{K}^{+}\left(\mathrm{K}_{\text {ATP }}\right)$ channels, in the mitochondria and sarcolemma, to $\mathrm{K}_{\mathrm{ATP}}$ channel openers (37). The results of this aforementioned study, which revealed that STVNa did not affect mitochondrial membrane potential, is in agreement with the consensus that STVNa does not solely activate $\mathrm{K}_{\mathrm{ATP}}$ channels (37), but rather makes channels more sensitive to endogenous channel openers. It has also been previously established that lactate, a product of anaerobic metabolism in the heart, activates $\mathrm{K}_{\text {АTP }}$ channels irrespective of high intracellular ATP levels $(38,39)$. The activation of mitochondrial and sarcolemmal $\mathrm{K}_{\text {ATP }}$ channels has been demonstrated to regulate intracellular $\mathrm{Ca}^{2+}$ homeostasis $(30,31)$. Therefore, the regulation of $\mathrm{Ca}^{2+}$ homeostasis by STVNa corresponds with its ability to sensitize $K_{\text {АTP }}$ channels to $K_{\text {АTP }}$ channel openers.

In conclusion, the current study demonstrated that STVNa prevents the development of cardiac hypertrophy, which is induced by Iso by preventing ROS generation, protecting mitochondrial function and regulating intracellular $\mathrm{Ca}^{2+}$ homeostasis. These results suggest that STVNa should be a potential therapeutic strategy against cardiac hypertrophy and heart failure in the future.

In the current study, the therapeutic effect of STVNa and the underlying mechanism by matching in vitro and in vivo experiments was defined. However, ex vivo experiments were not performed, which would provide another layer of tests for the present hypothesis. This could be viewed as a limitation of the present study although the in vitro and in vivo experiments match each other very well and strongly support the conclusions.

\section{Acknowledgements}

Not applicable.

\section{Funding}

The present study was supported by a grant from the 'Major Science and Technology Projects', Bureau of Science, Technology \& Information, Guangzhou City, 2013 (Category reference number 164; grant. no. 201300000051) and the National Natural Science Foundation of China (grant. no. 31300940). AJ was supported by the University of Nicosia Medical School. 


\section{Availability of data and materials}

The dataset used and/or analyzed during the current study are available from the corresponding author on reasonable request.

\section{Authors' contributions}

YC and WT designed the experiments. YC, HB, HS, FF, ZF and NL performed the experiments and YC, HS and AJ analyzed the data. AJ and YC wrote the manuscript. All authors read and approved the final manuscript.

\section{Ethics approval and consent to participate}

All animal experimental protocols complied with the Guide for the Care and Use of Laboratory Animals, published by the United States National Institutes of Health. The current study was approved by the Institutional Animal Research Committee of South China University of Technology.

\section{Patient consent for publication}

Not applicable.

\section{Competing interests}

The authors declare that they have no competing interests.

\section{References}

1. Hu H, Sun Xo, Tian F, Zhang H, Liu Q and Tan W: Neuroprotective effects of isosteviol sodium injection on acute focal cerebral ischemia in rats. Oxid Med Cell Longev 2016: 1379162, 2016.

2. Zhang H, Sun X, Xie Y, Zan J and Tan W: Isosteviol sodium protects against permanent cerebral ischemia injury in mice via inhibition of NF- $\kappa \mathrm{B}$-mediated inflammatory and apoptotic responses. J Stroke Cerebrovasc Dis 26: 2603-2614, 2017.

3. Zan J,Zhang H, Lu MY, Beng HM, Zhong KL, Sun XO and Tan W: Isosteviol sodium injection improves outcomes by modulating TLRs/NF- $\kappa \mathrm{B}$-dependent inflammatory responses following experimental traumatic brain injury in rats. Neuroreport 29: 794-803, 2018

4. Zhong KL, Lu MY, Liu F, Mei Y, Zhang XJ, Zhang H, Zan J, Sun XO and Tan W: Isosteviol sodium protects neural cells against hypoxia-induced apoptosis through inhibiting MAPK and NF- $\kappa$ B pathways. J Stroke Cerebrovasc Dis 28: 175-184, 2019.

5. Sun X, Yang Y, Xie Y, Shi X, Huang L and Tan W: Protective role of STVNa in myocardial ischemia reperfusion injury by inhibiting mitochondrial fission. Oncotarget 9: 1898-1905, 2017.

6. Liu Q, Hu H, Hu T, Han T, Wang A, Huang L, Tan Q and Tan W: STVNa attenuates right ventricle hypertrophy and pulmonary artery remodeling in rats induced by transverse aortic constriction. Biomed Pharmacother 101: 371-378, 2018.

7. Tang SG, Liu XY, Ye JM, Hu TT, Yang YY, Han T and Tan W: Isosteviol ameliorates diabetic cardiomyopathy in rats by inhibiting ERK and NF- $\mathrm{KB}$ signaling pathways. J Endocrinol 238 47-60, 2018.

8. Dini FL, Galeotti GG, Terlizzese G, Fabiani I, Pugliese NR and Rovai I: Left ventricular mass and thickness: Why does it matter? Heart Fail Clin 15: 159-166, 2019.

9. Bertero E and Maack C: Metabolic remodelling in heart failure. Nat Rev Cardiol 15: 457-470, 2018.

10. Haselhuhn LR, Brotman DJ and Wittstein IS: Heart failure guidelines: What you need to know about the 2017 focused update. Cleve Clin J Med 86: 123-139, 2019.

11. Tse J, Powell JR, Baste CA, Priest RE and Kuo JF: Isoproterenol-induced cardiac hypertrophy: Modifications in characteristics of beta-adrenergic receptor, adenylate cyclase, and ventricular contraction. Endocrinology 105: 246-255, 1979.
12. Morisco C, Zebrowski DC, Vatner DE, Vatner SF and Sadoshim J: Beta-adrenergic cardiac hypertrophy is mediated primarily by the beta(1)-subtype in the rat heart. J Mol Cell Cardiol 33: 561-573, 2001.

13. Osadchii OE: Cardiac hypertrophy induced by sustained beta-adrenoreceptor activation: Pathophysiological aspects. Heart Fail Rev 12: 66-86, 2007.

14. Nichtova Z, Novotova M, Kralova E and Stankovicova T: Morphological and functional characteristics of models of experimental myocardial injury induced by isoproterenol. Gen Physiol Biophys 31: 141-151, 2012.

15. Krenek P,Kmecova J,Kucerova D, Bajuszova Z, Musil P, Gazova A, Ochodnicky P, Klimas J and Kyselovic J: Isoproterenol-induced heart failure in the rat is associated with nitric oxide-dependent functional alterations of cardiac function. Eur J Heart Fail 11: 140-146, 2009.

16. Claycomb WC and Palazzo MC: Culture of the terminally differentiated adult cardiac muscle cell: A light and scanning electron microscope study. Dev Biol 80: 466-482, 1980.

17. Sergeeva IA and Christoffels VM: Regulation of expression of atrial and brain natriuretic peptide, biomarkers for heart development and disease. Biochem Biophys Acta 1832: 2403-2412, 2013.

18. Shin E, Ko KS, Rhee BD, Han J and Kim N: Different effects of prolonged $\beta$-adrenergic stimulation on heart and cerebral artery. Integr Med Res 3: 204-210, 2014.

19. Zhang GX, Kimura S, Nishiyama A, Shokoji T, Rahman M, Yao L, Nagai Y, Fujisawa Y, Miyatake A and Abe Y: Cardiac oxidative stress in acute and chronic isoproterenol-infused rats. Cardiovasc Res 65: 230-238, 2005.

20. Srivastava S, Chandrasekar B, Gu Y, Luo J, Hamid T, Hill BG and Prabhu SD: Downregulation of cuzn-superoxide dismutase contributes to beta-adrenergic receptor-mediated oxidative stress in the heart. Cardiovasc Res 74: 445-455, 2007.

21. Takimoto E and Kass DA: Role of oxidative stress in cardiac hypertrophy and remodeling. Hypertension 49: 241-248, 2007.

22. Vidal M, Wieland T, Lohse MJ and Lorenz K: $\beta$-Adrenergic receptor stimulation causes cardiac hypertrophy via a G $\beta \gamma /$ Erk-dependent pathway. Cardiovasc Res 96: 255-264, 2012.

23. Cha HN, Choi JH, Kim YW, Kim JY, Ahn MW and Park SY: Metformin inhibits isoproterenol-induced cardiac hypertrophy in mice. Korean J Physiol Pharmacol 14: 377-384, 2010.

24. Murphy MP: How mitochondria produce reactive oxygen species. Biochem J 417: 1-13, 2009.

25. Jovanović A: Cardioprotective signaling: Past, present and future. Eur J Pharmacol 833: 314-319, 2018.

26. Zhou B, Wu LJ, Li LH, Tashiro S, Onodera S, Uchiumi F and Ikejima T: Silibinin protects against isoproterenol-induced rat cardiac myocyte injury through mitochondrial pathway after up-regulation of sirt1. J Pharmacol Sci 102: 387-395, 2006.

27. Zhang Y, Xu J, Long Z, Wang C, Wang L, Sun P, Li P and Wang T: Hydrogen $\left(\mathrm{H}_{2}\right)$ inhibits isoproterenol-induced cardiac hypertrophy via antioxidative pathways. Front Pharmacol 7: 392, 2016.

28. Remondino A, Kwon SH, Communal C, Pimentel DR, Sawyer DB, Singh K and Colucci WS: Beta-Adrenergic receptor-stimulated apoptosis in cardiac myocytes is mediated by reactive oxygen species/c-Jun NH2-terminal kinase-dependent activation of the mitochondrial pathway. Circ Res 92: 136-138, 2003.

29. Eisner DA, Caldwell JL, Kistamás K and Trafford AW: Calcium and excitation-contraction coupling in the heart. Circ Res 121: 181-195, 2017.

30. Du Q, Jovanović S, Clelland A, Sukhodub A, Budas G, Phelan K, Murray-Tait V, Malone L and Jovanović A: Overexpression of SUR2A generates a cardiac phenotype resistant to ischaemia. FASEB J 20: 1131-1141, 2006.

31. Sukhodub A, Sudhir R, Du Q, Jovanović S, Reyes S and Jovanović A: Nicotinamide-rich diet improves physical endurance by up-regulating SUR2A in the heart. J Cell Mol Med 15: 1703-1712, 2011.

32. de Lucia C, Eguchi A and Koch WJ: New insights in cardiac $\beta$-adrenergic signaling during heart failure and aging. Front Pharmacol 9: 904, 2018.

33. Rengo G, Lymperopoulos A and Koch WJ: Future g protein-coupled receptor targets for treatment of heart failure. Curr Treat Options Cardiovasc Med 11: 328-338, 2009.

34. Bristow MR, Ginsburg R, Umans V, Fowler M, Minobe W, Rasmussen R, Zera P, Menlove R, Shah P, Jamieson S, et al: Beta 1and beta 2-adrenergic-receptor subpopulations in nonfailing and failing human ventricular myocardium: Coupling of both receptor subtypes to muscle contraction and selective beta 1-receptor downregulation in heart failure. Circ Res 59: 297-309, 1986. 
35. Dorn GW II and Force T: Protein kinase cascades in the regulation of cardiac hypertrophy. J Clin Invest 115: 527-537, 2005.

36. Bertero $\mathrm{E}$ and Maack $\mathrm{C}$ : Calcium signaling and reactive oxygen species in mitochondria. Circ Res 122: 1460-1478, 2018.

37. Fan Z, Wen T, Chen Y, Huang L, Lin W, Yin C and Tan W: Isosteviol sensitizes sarcKATP channels towards pinacidil and potentiates mitochondrial uncoupling of diazoxide in Guinea Pig ventricular myocytes. Oxid Med Cell Longev 2016: 6362812, 2016.

38. Jovanović S, Du Q, Sukhodub A and Jovanović A: M-LDH physically associated with sarcolemmal $\mathrm{K}_{\mathrm{ATP}}$ channels mediates cytoprotection in heart embryonic $\mathrm{H} 9 \mathrm{C} 2$ cells. Int J Biochem Cell Biol 41: 2295-2301, 2009.
39. Jovanović S, Du Q, Sukhodub A and Jovanović A: Dual mechanism of cytoprotection afforded by M-LDH in embryonic heart H9C2 cells. Biochim Biophys Acta 1793: 1379-1386, 2009.

(i) (9) This work is licensed under a Creative Commons Attribution-NonCommercial-NoDerivatives 4.0 International (CC BY-NC-ND 4.0) License. 